\title{
El analfabetismo en España. Un estudio a través del censo de población de 1877 \\ GLORIA ESPIGADO TOCINO
}

Finalizaba 1900, cuando el Boletín que publicaba la Institución Libre de Enseñanza recogía las opiniones que sobre el problema del analfabetismo en España tenía el catedrático de Farmacia y Medicina de la Universidad de Madrid, Federico Olóriz. Analizando los datos del censo de población de 1887 , este autor establecía una comparación entre el analfabetismo registrado en la población cuya edad oscilaba entre los 10 y los 35 años, es decir, aquellos que tuvieron que ser forzosamente escolarizados según ordenaba la Ley de Educación de Claudio Moyano, y los mayores de esa edad, que no conocieron los efectos de esa legislación. El resultado era la disminución, en más de un $8 \%$, de los analfabetos correspondientes al primer grupo de edad (1). Con ser importante dicha reducción, parecía insatisfactoria al comparar el porcentaje global de analfabetos en España (68\%, en 1887) con las cantidades inferiores de la mayoria de los países europeos: 35\% Irlanda, 36\% Francia, 42\% Bélgica, 44\% Austria, 57\% Hungría, 63\% Italia. Aunque siempre se podian poner ejemplos consoladores: $79 \%$ Portugal, 80\% Bulgaria, 93\% Rumanía (2). Sin embargo, Olóriz es el primero que advierte que nuestras estadísticas, al incluir en la elaboración de los porcentajes a los niños menores de 6 años que, por lo mismo, son incapaces de haber podido adquirir los conocimientos de lectura y escritura pertinentes, contribuyen a inflar por encima de la realidad nuestro número de analfabetos, resultándonos engañosa y perjudicial la comparación con otros países que sí atienden a esta particularidad.

(1) Federico Olóriz: "El analfabetismo en España", Boletin de la Institución Libre de Enseñanza (en adelante B.I.L.E.). Madrid, 1900, pp. 257-267 y 293-302. El indice de analfabetismo, especificado por este autor, entre los españoles de edad comprendida entre 10 y 35 años era de un $57,2 \%$, mientras que de 35 a 65 años se contabilizaba un $65,4 \%$.

(2) Ibidem., p. 260. 
En otro orden de cosas, y adentrándonos en nuestra propia reflexión sobre el particular, habrá que convenir que la cifra media de analfabetismo de cualquier país enmascara, oculta, situaciones muy dispares de unas zonas a otras de dicho espacio geográfico. En España, esta realidad la hemos podido constatar en función del reparto provincial del analfabetismo registrado en el censo de 1877. El mismo F. Olóriz advertía, efectuando un análisis más pormenorizado, que la distribución del analfabetismo de 1887 entre las provincias estaba lejos de constituir un mosaico irregular de realidades diferentes mezcladas unas con otras. Más bien, estaríamos ante grandes zonas de color uniforme donde la expresión del analfabetismo adquiriría homogeneidad. Los mapas que hemos elaborado a raíz de esta intuición a lo largo de este artículo demuestran cumplidamente la veracidad de esta hipótesis. Del mismo modo, aun siendo muy superior el analfabetismo de las mujeres en comparación con el de los hombres en España, habría que puntualizar a esta aseveración general, que existen zonas en el país donde la posición que ocupa el analfabetismo femenino se acerca a la situación que registra el otro sexo. No cabe duda, igualmente, que de la confrontación de los índices del analfabetismo rural y urbano resultará un saldo ampliamente positivo del primero respecto al cómputo que presenta el segundo. Por último, en el capítulo de las explicaciones, de la exposición de razonamientos que interpretan los fenómenos, el autor que nos está sirviendo como introductor al tema planteado cita, por este orden: la raza, la religión, las instituciones, la organización de la enseñanza y el ambiente social, como causas fundamentales que generan la situación de analfabetismo del país. Está claro que sin un estudio minucioso de las realidades escolares de cada zona, de los efectos que las condiciones económicas, sociales y mentales de la misma puedan producir en el proceso educativo, no se podrá obtener una respuesta acertada de las razones generales que explican el diferente grado de alfabetización alcanzado por un grupo homogéneo en el comportamiento de esta variable. Es aquí donde la historia local o regional, poco utilizada en estos temas hasta la fecha, puede cumplir un papel insustituible y definitivo para la comprensión de las diferencias interregionales apreciables en nuestro país. De forma que, analizando cada una de las partes, obtengamos una visión más compleja, quizá, pero más verdadera del todo, de la Historia del sistema educativo español, entendido no exclusivamente, como una sucesión de leyes y reglamentos oficiales, sino también, y fundamen- 
talmente, como las realizaciones prácticas en que se resuelve en cada localidad este conjunto de disposiciones generales (3).

Decir que España se encuentra durante todo el siglo XIX descolgada de la lista de aquellos países europeos que poseen un aceptable grado de alfabetización, dentro del conjunto de su población, sería poco. La realidad del analfabetismo en nuestro país no resiste comparación alguna con los avances que, en este campo, se aprecian en el resto del mapa europeo ya a mediados de siglo, y, lo que es más, la lenta evolución decreciente que experimentan estas cifras en España está lejos de representar los importantes progresos conseguidos por otros países que, en principio, partían de una situación bastante pareja. Hacia 1850, según datos de Carlo M. Cipolla (4), Europa podía dividirse, atendiendo a la consideración de la instrucción elemental de su población suceptible de recibir instrucción (mayores de 7 años, por lo general), en tres grupos de países cuyo porcentaje de analfabetismo iba de menor a mayor. (véase mapa).

El primero de éstos, o grupo A, comprendía a aquéllos en donde el número de los que no sabían leer ni escribir no superaba el $30 \%$, es decir, países con un nivel de población analfabeta bajo. Geográficamente hablando, este espacio se vinculaba, sin el menor género de dudas, con los llamados países nórdicos (Dinamarca, Finlandia, Noruega, Suecia e Islandia), a los que se sumaban también Holanda, Escocia, Suiza y Alemania. A mitad de siglo, los porcentajes medios de analfabetismo, del 30 al $50 \%$, continuaban respetando la mitad sur del continente y se situaban, en todos los casos, por encima de esta línea imaginaria.

(3) Escasos son todavía los trabajos que acometen el estudio zonal de las condiciones educativas de nuestro país. Para el siglo XIX podríamos citar algunos ejemplos: Hernández Díaz, J.M.: Educación y Sociedad en Béjar durante el siglo XIX, I.C.E.: Salamanca, 1983; Vega Gil, L.: Historia de la educación en Zamora. El nacimiento del sistema escolar. 1800-1850. Diputación Provincial de Zamora, 1986; Viñao Frago, A.: Historia de la educación en Murcia, Servicio de publicaciones de la Universidad de Murcia, 1983; Solá Gussinyer, P.: Cultura popular, éducació $i$ societat al Nord-Est Catalá (1887-1959), Collegi Universitari de Girona, 1983; Vicente Jara, F.: La Enseñanza primaria en Murcia en el siglo XIX, (1800-1857), Consejería de Cultura, Educación y Turismo. Murcia, 1989.

(4) Carlo M. Cipolla: Educación y desarrollo en Occidente. Ariel, Barcelona, $2^{2}$ ed. 1983. El conjunto europeo (menos Rusia) presenta un porcentaje de analfabetismo, hacia 1850, entre el 45 y el 50\%, porcentaje último que sirve a Cipilla para establecer la frontera entre los países, con aceptable grado de instrucción y aquellos que están dominados por la realidad del analfabetismo, p. 90. 


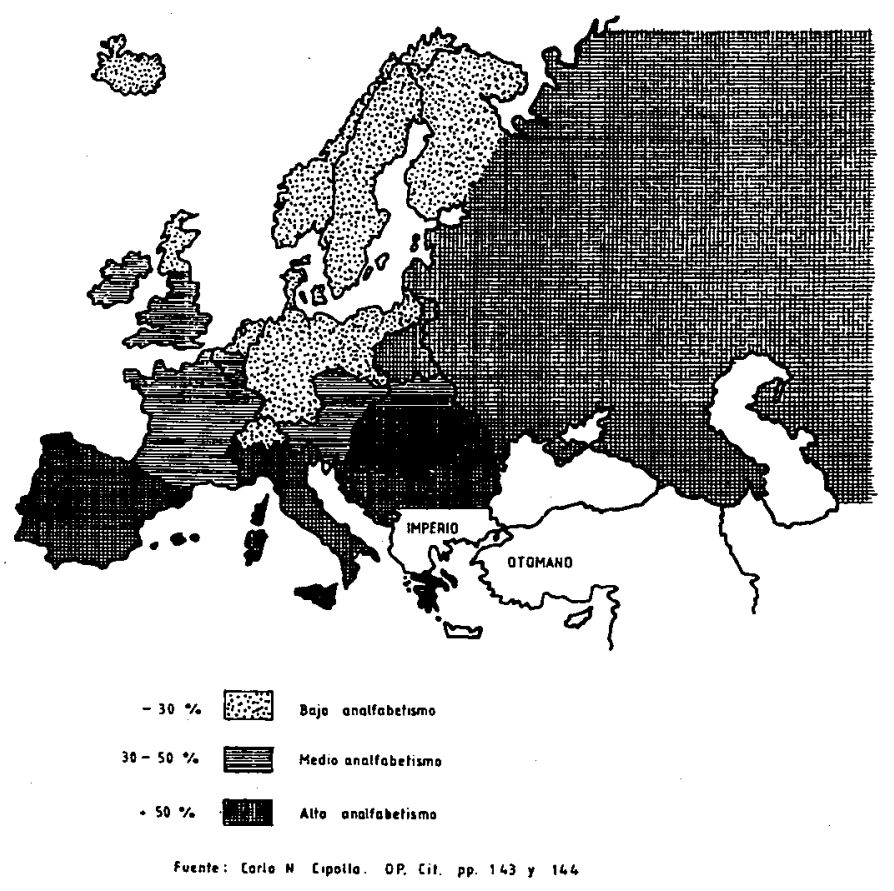

\section{Analfabetismo en Europa hacia 1850}

El Imperio Austriaco, Bélgica, Francia, Irlanda, Luxemburgo e Inglaterra (más Gales) se incluían en el grupo B de los países medios. La sorprendente ausencia del país pionero en acometer la Revolución Industrial (analizado siempre como modelo previo al proceso verificado posteriormente en el Continente) en el grupo de cabeza, quedaría suficientemente explicada, según Cipolla, por la peculiar relación coste-oportunidad que se establecería en los primeros estadios de la industrialización. Puesto que enviar un niño a la escuela significa renunciar a la oportnidad de un suplemento salarial ganado a costa de su trabajo, las buenas perspectivas laborales que acompañaban el avance y desarrollo de las industrias para el empleo de los niños redundaría en el aumento del coste-oportunidad de dar instrucción al conjunto de la población infantil, de modo que, en principio, este aumento global de la riqueza que sigue a la Revolución Industrial no se emplearía, al menos por lo que respecta a sus primeras fases, en mejorar el nivel de instrucción general de Inglaterra, la cual reflejaría en sus estadísticas cierto estancamiento en el momento de producirse su transformación económica. Sólo más adelante aprovecharía parte 
de su excedente en la fórmación e instrucción de la población obrera, en función de satisfacer la necesidad de especialización en el trabajo que requería su segundo estadio de industrialización. El tercer grupo de países (grupo C) supera el $50 \%$ de analfabetismo adulto y reúne a los que están menos desarrollados económicamente. Son los últimos en industrializarse en Europa y se sitúan en la mitad sur de la misma, a orillas del mar Mediterráneo. Bulgaria, Grecia, Hungría, Rumanía, Italia, España y Portugal, son integrantes del panorama más desolador de Europa. Nuestro particular interés, centrado, por supuesto, en la evolución experimentada por España, nos señala cuắn lejos se encontraba de alcanzar los mínimos de países como Francia que, en el año de 1872 , contabilizaba el $31 \%$ de población analfabeta nada más, o el escaso $12 \%$ de Prusia hacia 1871 , que le valía su inclusión entre los países de vanguardia en el proceso de difusión de la instrucción elemental. Por otro lado, si es el bajo índice de alfabetización lo que destaca y define la situación española dentro del contexto europeo de mediados de siglo, el segundo rasgo distintivo, sin duda alguna, va a ser la excesivamente lenta reacción emprendida con el objeto de invertir estos téminos, de modo que, aunque ciertamente la tasa porcentual inicia un apreciable descenso camino del fin de siglo, este esfuerzo alfabetizador se queda corto si lo comparamos con los logros que, por ejemplo Italia, país que en la década de los setenta ofrecía un panorama muy semejante al español, ha conseguido a comienzos del siglo XX. Así, en 1901, los italianos han rebajado al $48 \%$ su tasa, mientras España pierde ritmo y se descuelga aún más del cómputo que ofrecen los países desarrollados del norte europeo, registrando, solamente, un descenso muy moderado, hasta situarse en tomo al $56 \%$ en el censo de 1900 (teniendo en cuenta a los mayores de 10 años) (5).

Aunque estamos centrando nuestra atención en el análisis del siglo XIX europeo, es claro que, para la historiografía especializada en el ámbito de la educación, los factores que explican la evolución de una Europa escolarizada en su mayoría y por ende alfabetizada, han sido varios, y su influencia ha operado en un "tempus" histórico que podríamos calificar de larga duración. Básicamente existen dos

(5) Algunos porcentajes de analfabetismo de paises europeos, citados por Cipolla, para 1900 , serían:

Bélgica

Francia

Austria

Ibidem., p. 157.
$19 \%$, considerando a la población mayor de 10 años.

$18 \%$, considerando a la población mayor de 5 años.

$23 \%$, considerando a la población mayor de 10 años. 
corrientes cuyos argumentos no tienen por qué ser excluyentes entre sí, aunque, a veces, la radicalización de los postulados manifestados así lo dejen entrever, que apostarían por dos períodos diferenciados en la concienciación europea en torno a la instrucción. El primero de ellos, o primera revolución escolar, tendría su desarrollo en la Edad Moderna y vendría dada por intereses proselitistas de la Iglesia Reformada, lo que explicaría los evidentes adelantos de la Europa de la "religión del libro" (6). La otra línea de interpretación apreciaría una segunda y más importante revolución escolar derivada de los cambios cualitativos que introduce la acción revolucionaria de la burguesía, en donde la sustitución del privilegio estamental por el derecho del ciudadano de la nueva sociedad de clases naciente compromete al Estado en materia educativa y garantiza el acceso igualitario de todos al conocimiento (7). En el fondo de todo, ya sean consideradas la Iglesia o el Estado como los principales promotores de esta nueva sensibilidad educativa, no pueden dejarse a un lado otra serie de factores que explican el Take-off de la alfabetización europea. Auspiciado por el desarrollo del capitalismo a partir del Renacimiento, el despegue demográfico que éste conlleva ensancha las pirámides de población por su base, haciendo más patente ante la vista la cohorte de niños y de jóvenes, lo que provoca, a su vez, un replanteamiento de las necesidades del hombre en este período biológico, siendo la obra de Malthus tan sólo el ejemplo más conocido. Sobre la repercusión que la industrialización acarrea no vamos a insistir, remitiéndonos a lo dicho anteriormente, sólo apuntaremos que, las dos clases emergentes, burguesía y proletariado, y por razones diferentes, estarán interesadas en universalizar los conocimientos. La primera, por razones de rendimiento económico y profilaxis social; y la segunda, como medio de conseguir su emancipación. Por otro lado, podría citarse también el avance de los nacionalismos por lo que a orgullo de unidad, no sólo étnica sino también cultural, se refiere. Por último, el desarrollo de la ciencia en general y de las humanidades en particular consagran un interés creciente, que ya despuntaba en los escritos roussonianos, de la paidología (8).

(6) Furet, F. y Ozouf, J.: Lire et écrire. L'alphabétization des français de Calvin a Jules Ferry. París, Editions de Minuit, 1977, 2 Vol.

(7) Hobsbawm, E.J., "La carrera abierta al talento", en Las Revoluciones burguesas. Labor, Barcelona, 6ㄹ ed. 1976, Vol. II, pp. 325-357.

(8) Escolano Benito, A.: "Historia de la escolarización e Historia Social", en Educación y Sociedad en la España Contemporánea (1808-1970). Valencia, 1983, pp. 979-998. 
Huelga decir que esta visión de conjunto a la que estamos sometiendo a los distintos países de Europa en su evolución hacia la alfabetización de sus habitantes no puede perder de vista, en ningún caso, las profundas diferencias interregionales que se aprecian puertas adentro de estos mismos, de forma que los porcentajes comparativos usados hasta aquí enmascaran, ocultan, como medias nacionales que son, situaciones internas muy dispares. Del mismo modo, llegado el caso, sería conveniente afinar aún más en las distintas escalas espaciales, considerando, oportunamente, tanto el grado de alfabetización urbana como el estado de las distintas comarcas rurales, en donde una localización geográfica desfavorable, unos insuficientes recursos económicos, etc..., pueden alejar irremediablemente a una comunidad campesina del circuito de la cultura o de la simple instrucción elemental. Estos desajustes internos en el nivel de conocimientos primarios entre los distintos habitantes de un país necesitan una explicación, pensamos, partiendo de la propia realidad económica y social de la región de la que forman parte. A nadie se le escapa que la Italia del norte es la primera y única región del país en la que resulta ser una realidad el proceso industrializador, al mismo tiempo que el sur es una carencia total en este sentido. De otra parte, la Prusia oriental y la Posnania polacas son eminentemente campesinas, donde el desigual reparto de la propiedad pone al servicio de unos pocos, los junkers, a la mayoría de la población polaca inculta. En el caso de España, el análisis de los distintos porcentajes de analfabetismo provincial, según el censo de 1877 (9), han revelado algo más que los simples tópicos que sobre esta materia se mantenían. Estos hacen referencia a dos hechos básicamente: el primero, que la España alfabetizada mayoritariamente se

(9) Es obvio que para la contemporaneidad los censos de población son una fuente de inestimable valor para sacar consecuencias cuantitativas como las que aquí traemos. Las dudas que podemos tener sobre la fiabilidad de los datos que aportan siempre serán preferibles a la escasez de fuentes rigurosas con las que cuentan otras etapas históricas. Por ejemplo, para el estudio de la alfabetización en la modernidad cada vez son más utilizados la documentación notarial, concretamente las firmas estampadas en los testamentos. Trabajan con este tipo de fuentes: Marcos Alvarez, F. y Cortés Cortés, F., Educación y Analfabetismo en la Extremadura Meridional (siglo XVII). Servicio de Publicaciones de la Universidad de Extremadura, Cáceres, 1987 y $\mathrm{M}^{\mathrm{a}}$ José de la Pascua Sánchez:"Aproximación a los niveles de alfabetización en la provincia de Cádiz: las poblaciones de Cádiz, El Puerto de Santa María, Medina Sidonia y Alcalá de los Gazules", en Trocadero, Revista de Historia Moderna y Contemporánea de la Universidad de Cádiz, n 1, 1989, pp. 51-67. 
sitúa en la mitad norte de la península; y el segundo, de forma inversa, que la España eminentemente inculta ocupa la mitad sur de nuestro país. Sin embargo, pensamos que estas expresiones tan genéricas son suceptibles de una mayor precisión en función de las cifras porcentuales que aparecen en la primera tabla de este trabajo, la cual se acompaña gráficamente de su respectivo mapa.

\section{Analfabetismo provincial en España hacia 1877.}

$\begin{array}{llllllll}\text { Provincias } & \text { Mas. } & \text { Fom. } & \text { Total } & \text { Provincias } & \text { Mas. } & \text { Fom. } & \text { Total } \\ \text { ALAVA } & 19,93 & 41,31 & 30,39 & \text { ZARAGOZA } & 57,85 & 80,86 & 69,48 \\ \text { MADRID } & 26,68 & 50, & 38,48 & \text { PONTEVEDRA } & 45,98 & 86,59 & 69,70 \\ \text { PALENCIA } & 19,94 & 56,66 & 38,53 & \text { CACERES } & 59,71 & 81,54 & 70,43 \\ \text { CANTABRIA } & 18,21 & 55,67 & 38,70 & \text { HUESCA } & 57,46 & 86,20 & 71,59 \\ \text { BURGOS } & 19,33 & 50,20 & 39,99 & \text { BADAJOZ } & 65,50 & 79,11 & 72,22 \\ \text { SEGOVIA } & 26,61 & 59,24 & 41,44 & \text { TERUEL } & 57,68 & 87,59 & 72,85 \\ \text { VALLADOLID } & 29,86 & 61,77 & 46,17 & \text { TARRAGONA } & 63,44 & 82,14 & 72,90 \\ \text { SORIA } & 22,43 & 69,58 & 47,20 & \text { CORUNA } & 55,59 & 86,59 & 73,32 \\ \text { NAVARRA } & 42,03 & 57,95 & 49,86 & \text { CORDOBA } & 68,42 & 80,11 & 74,30 \\ \text { RIOJA } & 35,28 & 63,88 & 50,01 & \text { CIUDADREAL } & 65,66 & 83,62 & 74,64 \\ \text { GUIPUZCOA } & 48,07 & 52,21 & 50,15 & \text { LERIDA } & 64,10 & 86,08 & 75,06 \\ \text { VIZCAYA } & 40,59 & 60, & 50,45 & \text { LUGO } & 57,13 & 91,98 & 75,82 \\ \text { LEON } & 29,20 & 75,13 & 53,41 & \text { ORENSE } & 59,16 & 91,13 & +76,22 \\ \text { SALAMANCA } & 38,37 & 62,29 & 54,10 & \text { BALEARES } & 68,65 & 84,47 & 76,89 \\ \text { ZAMORA } & 33, & 76,87 & 54,61 & \text { JAEN } & 72,24 & 79,83 & 77,71 \\ \text { BARCELONA } & 44,51 & 68,73 & 56,80 & \text { MURCIA } & 71,47 & 85,25 & 78,44 \\ \text { AVILA } & 42,51 & 71,22 & 56,98 & \text { VALENCIA } & 73,13 & 84,03 & 78,66 \\ \text { ASTURIAS } & 36,40 & 74,57 & 57,82 & \text { CANARIAS } & 70,01 & 82,15 & 79,83 \\ \text { GUADALAJARA } & 40,14 & 76,51 & 57,92 & \text { ALBACETE } & +71,62 & 87,87 & 79,84 \\ \text { CADIZ } & 60,54 & 68,41 & 64,38 & \text { ALICANTE } & 73,77 & 85,63 & 79,89 \\ \text { GERONA } & 54,25 & 81,37 & 67,84 & \text { MALAGA } & 75,51 & 84,20 & 79,97 \\ \text { SEVILLA } & 62,78 & 73,40 & 68,08 & \text { CASTELLON } & 73,46 & 89,38 & \mathbf{8 1 , 5 6} \\ \text { CUENCA } & 55,57 & 64,67 & 68,42 & \text { ALMERIA } & 76,08 & 89,55 & 83,22 \\ \text { HUELVA } & 62,08 & 76,37 & 68,79 & \text { GRANADA } & 80,49 & 89,14 & 84,88 \\ \text { TOLEDO } & 59,53 & 78,90 & 69,09 & \text { ESPANA } & 54,70 & 77,26 & 66,30\end{array}$

Fue nte: Elaboración propia a partir del Censo de población de España de 1877.
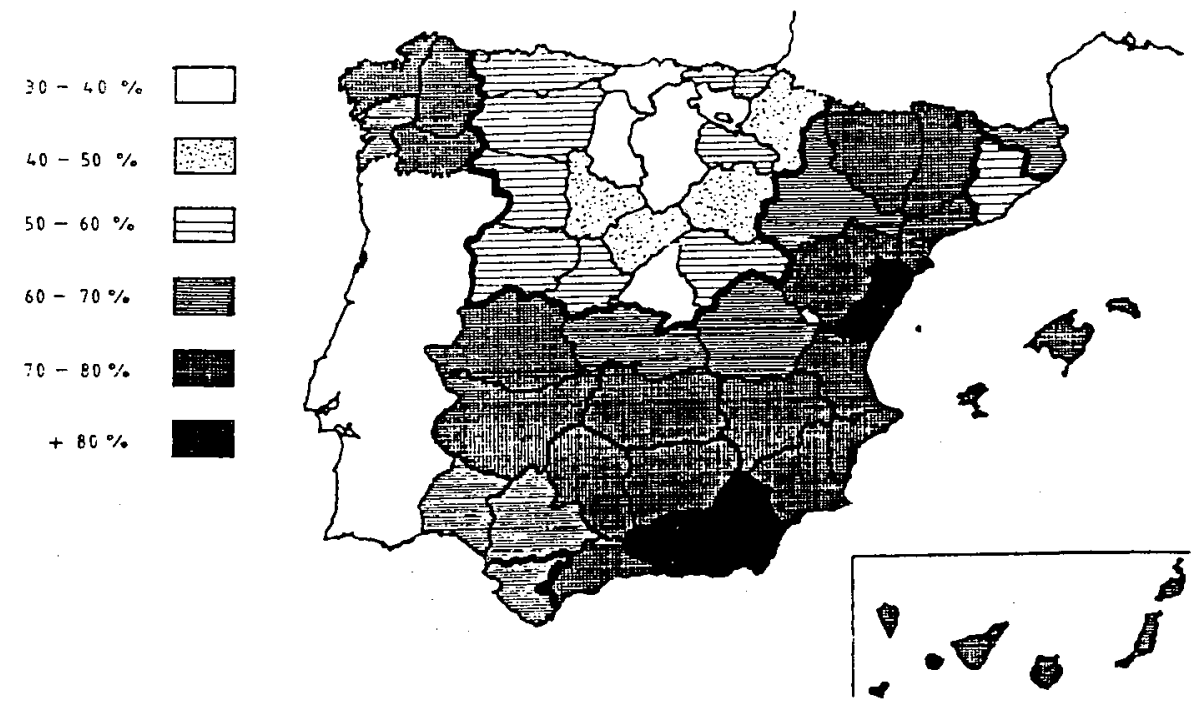
Tenemos que advertir que estos porcentajes recogen la población analfabeta de España de edad superior a 7 años, edad en la que normalmente solía comenzar la escolarización, total o considerada por sexos, referidos, no al conjunto demográfico del país, incluyendo a los menores de 7 años, como hace el censo de 1877, sino descontando, precisamente, del cómputo general a esta población infantil mencionada. De este modo, se sigue mucho más de cerca el espíritu que inspira la confección del censo de la población analfabeta de este año, que inexplicablemente se traiciona en el mismo al hallar los porcentajes de población sin instruir teniendo en cuenta el total de la población de España, sin descontar, en este último apartado, a los que por edad aún no han sido escolarizados, es decir, sin restar a los menores de 7 años. Las diferencias entre las tasas de analfabetismo en España, que operan con el conjunto de la población inculta sobre el total de habitantes, y ésta, que elaboramos nosotros partiendo de la premisa de que hay que descontar a la población que, por su corta edad, no está en condiciones aún de aprender a leer y a escribir, no son, en modo alguno, despreciables. En el primer caso estarían la totalidad de los autores consultados, los cuales contemplan para 1877 el porcentaje del $72 \%$ de población de ambos sexos analfabeta en España, mientras que, según nuestros datos, hechas las puntualizaciones de edad pertinentes, estimamos en un $66.3 \%$ (cifra sensiblemente inferior a la anterior) a aquella población que estando en condiciones de adquirir instrucción no la posee todavía en ese año. Sólo de esta forma se consigue ser consecuente con los límites que impone la edad infantil en el acceso a la instrucción, al mismo tiempo que se obtienen cifras que pueden ser comparadas con las que Cipolla ofrece para el conjunto europeo siguiendo este mismo criterio (10).

La España que posee porcentajes inferiores a la media europea de mitad de siglo (50\%, exceptuando Rusia), se reduce a sólo 9 provincias: Alava, Madrid, Palencia, Cantabria, Burgos, Segovia, Valladolid,

(10) Por citar algunos ejemplos de autores que utilizan el dato porcentual global que arroja el censo de 1877, sin cuestionarse la conveniencia de reducir a los más jóvenes (hasta los 7 años) tendríamos a Artola, M.: La Burguesía Revolucionaria (1808-1874), Alfaguara V, Alianza; Madrid, 1981 ( $8^{a}$ ed.), p. 281; Martinez Cuadrado, M.: La Burguesía Consenvadora (1874-1931), Alfaguara VI, Alianza, Madrid, 1981 (7ª ed.), p. 124; Mayordomo Pérez, A.: Educación y cuestión obrera en la España Contemporánea, Nau Llibres, Valencia, 1981, p. 41; Puelles Benítez, M.: Educación e ideología en la España contemporánea (1867-1975), Politeia, Labor, Barcelona, 1980, p. 311. Nótese que algunas de estas obras, fundamentalmente las dos primeras, son de una notable divulgación. 
Soria y Navarra. Otras tres, La Rioja, Guipúzcoa y Vizcaya, rozan con sus cifras el límite de alfabetización media (30-50\% de analfabetos) que Cipolla, en el cuadro que realiza para Europa, denomina grupo B. Por tanto, podemos decir que, a casi un cuarto de siglo de las estimaciones que hace este autor para la Europa de 1850, nuestro país no ha logrado que alguna de sus provincias se equipare al conjunto de países de alto nivel de alfabetización (siempre por debajo del $30 \%$ de personas que no saben ni leer ni escribir), si exceptuamos el porcentaje, ciertamente notable, de la provincia de Alava (30,39\%). Como un cinturón envolvente a estas provincias y siempre circunscrito a la mitad norte peninsular, tal y como predicen, más que demuestran, las afirmaciones que sobre el tema se han hecho, localizamos el conjunto provincial que dentro de punto de mira hispano posee los tantos por ciento de analfabetismo más moderados. En todos los casos, éstos se encuentran por debajo del índice medio nacional de ese año: 66,3\% (trazo grueso del mapa). Bordean por el sur y el oeste a todas las ya descritas las provincias de Guadalajara, al este de la capital; Avila, al oeste de la misma, y siguiendo un corredor hacia el norte, Salamanca, Zamora, León y Asturias. Frente a este bloque compacto que conforman estas provincias con el conjunto anterior, en lo que podemos definir como un oasis de importante alfabetización, la provincia de Barcelona brilla con luz propia dentro de este grupo. Se trata de la provincia cuya capital y zonas adyacentes pueden ser consideradas a estas alturas de siglo como la única zona medianamente industrializada del país y, dado su evidente aislamiento del conjunto anterior, debemos pensar que es a esta circunstancia a la que debe su menor porcentaje de analfabetismo, aun teniendo en cuenta la particular relación coste-oportunidad que conlleva este proceso de industrialización, que no puede obviar, por otro lado, que espacios castellanos fuera de toda sospecha industrial estén por delante de ella en dicho concepto. El otro foco uniprovincial, situado en el extremo suroeste peninsular, que como Barcelona posee un porcentaje ligeramente inferior a la media nacional es Cádiz, con un $64,38 \%$. No se nos oculta la posibilidad de enlazar esta realidad tan sorprendente dentro del marco geográfico que la rodea, inmerso, sin ningún género de dudas, en lo que podríamos denominar la España inculta del siglo XIX, más bien con esplendores pasados antes que con esperanzas de futuro para la provincia gaditana y su capital. Bastante atrás quedan, para la ciudad gaditana y su entorno provincial más inmediato, los buenos negocios y las rápidas fortunas subsiguientes labradas en el comercio marítimo 
Posiblemente, los excedentes de este capitalismo comercial financiarían; finalmente, actividades menos relacionadas con la productividad y sí con el desarrollo cultural y espiritual de su población. Perdidas estas buenas perspectivas económicas a raíz de la emancipación de las colonias, subsistiría, empero, esta preocupación por los temas educativos a la que más bien debe la provincia su moderada cifra de alfabetización en 1877, a pesar de que la decadencia económica parece un hecho incontestable (11). Retomando el hilo de la explicación, diremos que no es nada fácil el análisis del desarrollo de la instrucción elemental en el primer gran sector provincial que comparte o reduce las cifras de Barcelona o Cádiz. Madrid posee el efecto, nada despreciable, de la capitalidad del reino, en donde la concentración del funcionariado, gran parte de las empresas editoras, de la prensa, de los centros de enseñanza en sus diferentes grados, etc... provoca, a buen seguro, unas mejores condiciones para la divulgación de los conocimientos. Sin embargo, para el resto del conjunto, mucho nos tememos que las explicaciones del fenómeno se muevan dentro del terreno de las hipótesis y conjeturas, cuya demostración requeriría una profundización y conocimientos mayores sobre la realidad económico-social y también, quizá, sobre los hábitos mentales desarrollados en estas provincias. Este problema sólo se resolvería, a nuestro juicio, abordando localmente este tema tan poco estudiado en España. De poco nos sirve la conexión del desarrollo industrial vasco con el considerable nivel de alfabetización de la zona. Probablemente, esta realidad sea anterior al fenómeno de Take-off siderúrgico de las provincias vascas

(11) La relación de la actividad comercial con un elevado indice de alfabetización parece seguro, cuando comprobamos que la responsabilidad casi absoluta de la moderada tasa de analfabetismo provincial gaditano corre a cargo de la reducción relativa que provoca el excelente grado de instrucción de las poblaciones más comprometidas con el intercambio mercantil, frente al panorama desolador que presentan pueblos del interior provincial, especialmente los de la zona serrana. Al respecto, ver Gloria Espigado Tocino: "Clasificación socioprofesional y analfabetismo en Puerto Real hacia 1873", en Gades, Educación y educadores en Cádiz, Homenaje al profesor Juan Benvenuty Morales, №18, Diputación Provincial de Cádiz, 1988, pp. 233-249. El propio Federico Olóriz se hacía eco de este particular fenómeno que diferenciaba claramente el comportamiento de los núcleos poblacionales gaditanos más importantes de su entorno: "Suele ocurrir en algunas naciones del nuevo continente y en ciertas grandes colonias, que las capitales contrastan extraordinariamente con el resto de la población, tanto que podrían compararse por su cultura relativa a focos luminosos cercados de profundísimas tinieblas... en nuestra patria, donde mayor se observa... es en Cádiz, donde el analfabetismo del campo supera en un $32 \%$ el de las ciudades", Op. Cit., p. 267. 
y escasamente, éste último, pudo tener serios efectos en esta cuestión, por estas fechas, si consideramos que el boom de la industria de extracción y transformación del hierro se sitúa a partir de la década de los ochenta-noventa, en definitiva, a finales del siglo pasado. En el mismo terreno especulativo nos movemos a la hora de apuntar otro factor que pudo incidir en lo que podríamos calificar como una cierta democratización de la enseñanza primaria en estas provincias de alto nivel de alfabetización. Considerando el carácter eminentemente rural de la actividad económica de España, y los distintos rasgos socioeconómicos que imprimen en cada región las diferentes modalidades de reparto de propiedad, ino sería aventurado pensar, como primera hipótesis de trabajo, que las zonas más alfabetizadas se relacionan con los núcleos donde predominan las tendencias medias de la propiedad, al mismo tiempo que el fenómeno se invierte en los dominios del minifundismo (Galicia) y del latifundismo (mitad sur peninsular)?. Dicho esto con todas las reservas, conscientes de que esta hipótesis requiere una verificación más profunda de la que aquí podemos ofrecer.

Sobrepasando ya el índice medio nacional, y a distancia más que considerable de las cifras que manejábamos para los países industrializados de Europa, encontramos a las 29 provincias españolas que restan por analizar. Los rasgos más sobresalientes de este espacio son los que apuntamos seguidamente. En primer lugar, destaca el foco noroccidental de la península, la región gallega, que se encuentra separada del resto de sus homólogas en esta cuestión por el bloque provincial compacto que posee los índices más bajos. El minifundismo, la insuficiente explotación de los recursos, la ausencia de actividad industrial, el tradicional recurso a la emigración, en definitiva, el atraso económico secular de esta región, podrían utilizarse como argumentos explicativos de esta situación. En segundo término, y tratándose de la principal novedad que nos interesa apuntar dentro de esta investigación eminentemente cuantitativa, decir que la línea divisoria desde donde se establece el dominio del analfabetismo español en sus índices máximos no coincidiría, como tópicamente se hace observar, con una horizontal que nos separa la mitad norte de la mitad sur peninsular, sino más bien con una diagonal que, salvo los casos excepcionales de Barcelona y Cádiz ya comentados, diseccionaría todo el espacio suroriental, mediterráneo, de nuestro país. Con porcentajes comparables, e incluso superiores, a los gallegos, figura toda la franja que va desde las provincias fronterizas a los Pirineos (Huesca: 71,59\% y Lérida: $75 \%$ ), hasta aquellas que se abren camino en el sur hacia el Atlántico y la frontera con Portugal (Sevilla: 68,08\%, Huelva: 68,79\% y Badajoz: $72,22 \%)$ pasando, siempre en este corredor transversal, por Córdoba: $74,3 \%$, Ciudad Real: 74,64\%, Toledo: 69,09\%, Cuenca: 64,48\%, Teruel: 
$72,85 \%$, Zaragoza: $69,48 \%$ y Tarragona: $72,9 \%$. Por último, reafirmando esta localización sureña y levantina de las regiones menos alfabetizadas de España, con más de las tres cuartas partes de su población mayor de 7 años sumida en el analfabetismo, tenemos las provincias que componen la Andalucía oriental (Málaga: 79,97\%, Jaen: 77,71\%, Almería: $83,22 \%$ y Granada: $84,88 \%$, con el índice más alto de toda España); la antigua región de Murcia (Albacete: $79,84 \%$ y Murcia: $78,44 \%$ ) y todo el reino de Valencia (Alicante: $79,89 \%$, Valencia: $78,66 \%$ y Castellón: $81,56 \%$ ), así como las islas Baleares $(76,89 \%)$ y Las Canarias $(79,83)$ incluidas también en este apartado, emulando otros comportamientos insulares europeos (12).

Al considerar ahora los porcentajes de analfabetismo, con la misma exclusión de los menores de 7 años efectuada anteriormente, en las distintas capitales de provincia, observamos como, lógicamente, todas las variables socio-económicas que entran en juego en el modo de vida urbano reducen considerablemente las cantidades manejadas.

\section{Analfabetismo en las capitales provinciales de España hacia 1877:}

\begin{tabular}{|c|c|c|c|c|c|c|c|}
\hline Capitales & Mas. & Fom. & Total & Capitales & Mas. & Fem. & Tota \\
\hline $\begin{array}{l}\text { VITORIA } \\
\text { MADRID }\end{array}$ & $\begin{array}{l}15,04 \\
16,93\end{array}$ & $\begin{array}{l}30,76 \\
41,43\end{array}$ & $\begin{array}{l}22,78 \\
29,73\end{array}$ & $\begin{array}{l}\text { ORENSE } \\
\text { TOLEDO }\end{array}$ & $\begin{array}{l}31,77 \\
38,99\end{array}$ & $\begin{array}{l}63,51 \\
61,13\end{array}$ & $\begin{array}{l}48,98 \\
58,99\end{array}$ \\
\hline BURGOS & 15,31 & 47,06 & 30,72 & TARRAGONA & 40,74 & 61,02 & 50.43 \\
\hline BILBAO & 22,58 & 40.13 & 32.01 & ZARAGOZA & 38,22 & 63,54 & 51,02 \\
\hline SEGOVIA & 18,85 & 44,52 & 32,46 & CIUDAD REAL & 43,27 & 65,42 & 53,8 \\
\hline PAMPLONA & 28,60 & 40,40 & 34,29 & BADAJOZ & 49,23 & 62,77 & 55,67 \\
\hline LEON & 17,85 & 49,91 & 34,73 & HUELVA & 50,30 & 61,15 & 55,8 \\
\hline SANTANDER & 19,65 & 48,10 & 35,45 & CORDOBA & 49,99 & 64,64 & 57,3 \\
\hline SORIA & 17,91 & 50,25 & 35,94 & LUGO & 39,73 & 73,29 & 57,5 \\
\hline AVILA & 23,66 & 49,79 & 37,51 & CACERES & 49,13 & 69,76 & 58,7 \\
\hline SALAMANCA & 23, & 50,03 & 37,75 & TERUEL & 44,57 & 73,48 & 59,5 \\
\hline VALLADOLID & 34,44 & 51,93 & 38,41 & ST.CRUZ.TEN. & 53,48 & 64,34 & 59,5 \\
\hline BARCELONA & 25,09 & 50,81 & 38,43 & ALICANTE & 51,20 & 71,22 & 61,8 \\
\hline PALENCIA & 22,18 & 53,25 & 38,72 & PALMA & 51,21 & 71 & 61,8 \\
\hline CADIZ & 32,35 & 47,04 & 40,13 & LERIDA & 54,20 & 72,72 & 63.5 \\
\hline GUADALAJARA & 28,20 & 51,67 & 40,53 & JAEN & 59,09 & 69,90 & 64,5 \\
\hline S. SEBASTIAN & 38,35 & 44,29 & 41,43 & PONTEVEDRA & 44,32 & 79,80 & 64,7 \\
\hline GERONA & 25,68 & 58,48 & 42,38 & VALENCIA & 63,41 & 68,14 & 65,8 \\
\hline LOGROÑO & 32,49 & 52,31 & 42,63 & MALAGA & 58,58 & 73,63 & 66,8 \\
\hline CUENCA & 34,21 & 53,85 & 44,63 & GRANADA & 60,62 & 73,43 & 67,3 \\
\hline OVIEDO & 29,36 & 59,38 & 45,55 & ALBACETE & 59,51 & 77,16 & 68,4 \\
\hline CORUÑA & 32,72 & 56,07 & 46,02 & MURCIA & 60,29 & 83,74 & 71,6 \\
\hline ZAMORA & 35,94 & 58,52 & 47,83 & ALMERIA & 63,69 & 79,78 & 72,3 \\
\hline SEVILLA & 39,39 & 56,38 & 48,29 & CASTELLON & 66,96 & 81,95 & 74,7 \\
\hline HUESCA & 36,54 & 59,94 & 48,89 & ESPAÑA & 37,16 & 57,63 & 47,8 \\
\hline
\end{tabular}

Fuente: Elaboración propia a partir del Censo de la población de España de 1877.

(12) Estado del analfabetismo italiano por zonas geográficas hacia 1871: Norte, 54\%; Centro, 75\%; Sur, $84 \%$ e Islas, $86 \%$. Porcentajes de Svimez, Statisiche sul Mezzogiorno d'Italia. Roma, 1954, p. 770, cifr. Cipolla, C.M., Op. Cit., p. 97. 

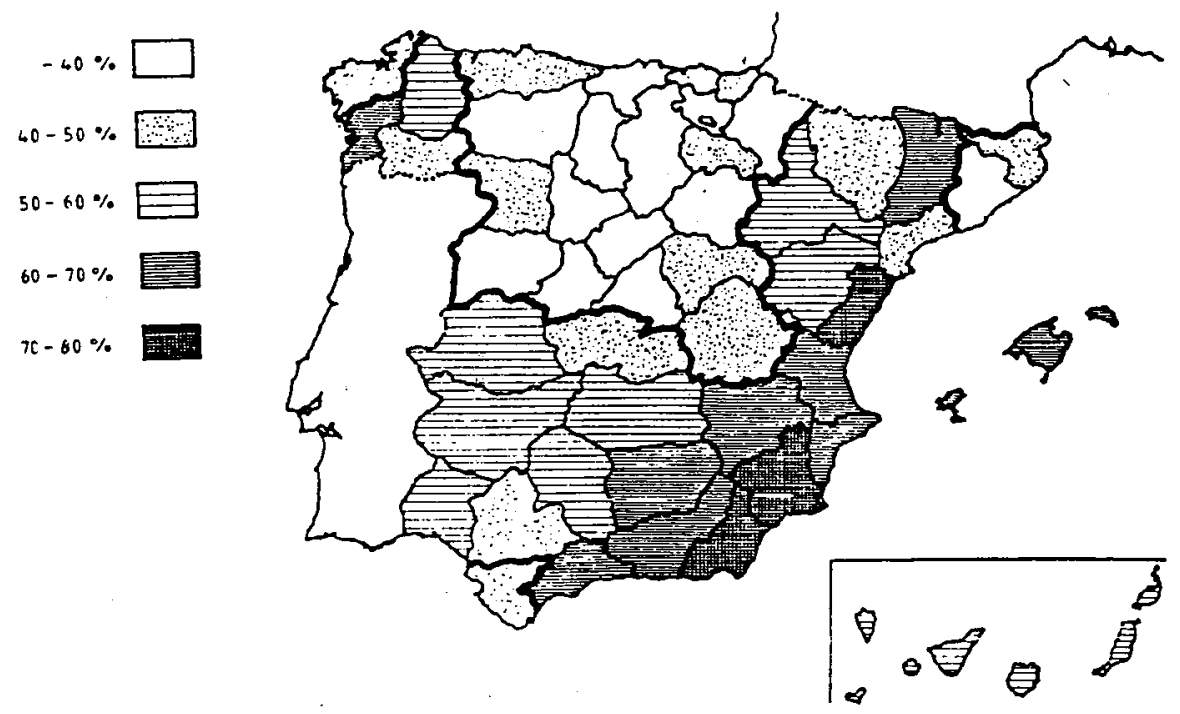

Teniendo en cuenta esta primera puntuálización, no se nos escapa, sin embargo, la exacta coincidencia geográfica que se da entre la ubicación de las capitales de provincia más alfabetizadas (mitad norte peninsular, foco barcelonés y foco gaditano, todos por debajo de la media nacional), con los conjuntos provinciales más instruidos vistos en las páginas anteriores; así como, también, es un hecho evidente la correspondencia entre las ciudades menos favorecidas en la instrucción de sus habitantes y aquella región suroriental que presentaba el mayor grado de analfabetismo provincial. Pero analicemos esta realidad por partes. En primer lugar, hay que destacar que solamente existen dos ciudades en España que rebajen el porcentaje límite de los países más desarrollados de Europa citados por Cipolla (menos del $30 \%$, recordemos), estas son: Madrid, con $29,73 \%$ y Vitoria, con $22,78 \%$, reafirmando su posición de vanguardia en el proceso de alfabetización del país. A continuación tenemos presente al conjunto urbano que se sitúa por debajo del límite de analfabetismo considerado como moderado ( $50 \%$ ), diferenciando aquellas capitales de provincia que no sobrepasan la media nacional registrada en 1877 que alcanza casi un $48 \%$. Dentro de esta tasa de analfabetismo ciudadano quedó expresada, con antelación, la coincidencia espacial existente con las áreas de analfabetismo regional. Sin embargo, hay que señalar que a este apartado se suman ciertas capitales que parecen desmarcarse un tanto del comportamiento medio provincial (proporcionalmente más elevado), para incluirse en el grupo que está por debajo de la 
media de las capitales de la nación. Con todo, no se registran sorpresas excesivas, ya que dichas capitales se reducen a los casos de La Coruña $(46,02 \%)$, Cuenca $(44,63 \%)$ y Gerona $(42,38 \%)$. Pasando al polo opuesto, al de las capitales con porcentajes máximos de analfabetismo, podemos apuntar que existe un espacio geográfico en nuestra península dentro del cual se podría hablar de analfabetismo integral, al coincidir, plenamente, el atraso provincial, computado en este sentido, con las cifras de analfabetismo de la capital de la provincia correspondiente, en todo caso siempre superior al $60 \%$. Esta región, a la que pertenecen Pontevedra, como único exponente gallego en esta ocasión, Palma de Mallorca y Lérida, incluye el conocido grupo de provincias de la Andalucía oriental, antigua región de Murcia y todo el Levante español, portador de las cantidades máximas. Entre ambos espacios de máximos y mínimos se encuentran las nueve capitales de transición que oscilan entre el 50 y el $60 \%$. Es decir, aunque en todo momento nos encontramos con tantos por ciento sensiblemente inferiores a los del conjunto provincial, tal y como corresponde a los medios y recursos que se concentran en nuestras principales ciudades, sin embargo, estas capitales se identifican, proporcionalmente, con la distribución geográfica del analfabetismo español descrita a través del primer mapa que incluíamos en nuestro trabajo.

Si consideramos ahora la variable en atención al sexo, en líneas generales, no resulta aventurado decir que la historia de la alfabetización de la población española en el siglo XIX comprende la paulatina extensión de la instrucción primaria elemental en el conjunto de la población masculina, mientras que, por el contrario, se mantienen en un nivel alto las cifras de analfabetismo femenino. Aunque la reducción intercensal registrada entre las mujeres que no saben leer ni escribir sea importante, incluso superior a la experimentada por los varones en el mismo período, no nos debe engañar este dato, ya que tenemos que caer en la cuenta de que partimos de un volumen global de analfabetismo muy superior al que presentan estos últimos, de unas cifras que casi incluyen la totalidad del censo femenino mayor de siete años, y desde donde la evolución, por mínimas que sean las mejoras emprendidas en estas fases iniciales de escolarización, siempre es más sencilla. Desde luego, considerando que el analfabetismo medio nacional femenino en 1877 es de un 77,26\%, y teniendo en cuenta, además, que en no pocas provincias (al menos la mitad) se sobrepasa el porcentaje del $80 \%$, no es difícil concluir que la alfabetización de la mujer en España es un fenómeno, cuando menos, del 
siglo XX (13). Por otro lado, la media de analfabetismo masculino, salvando las disparidades internas de las que luego hablaremos, se sitúa dentro de unos límites moderados que rondan el $54 \%$ de los varones mayores de 7 años.

\section{Analfabetismo provincial masculino Analfabetismo provincial femenino}
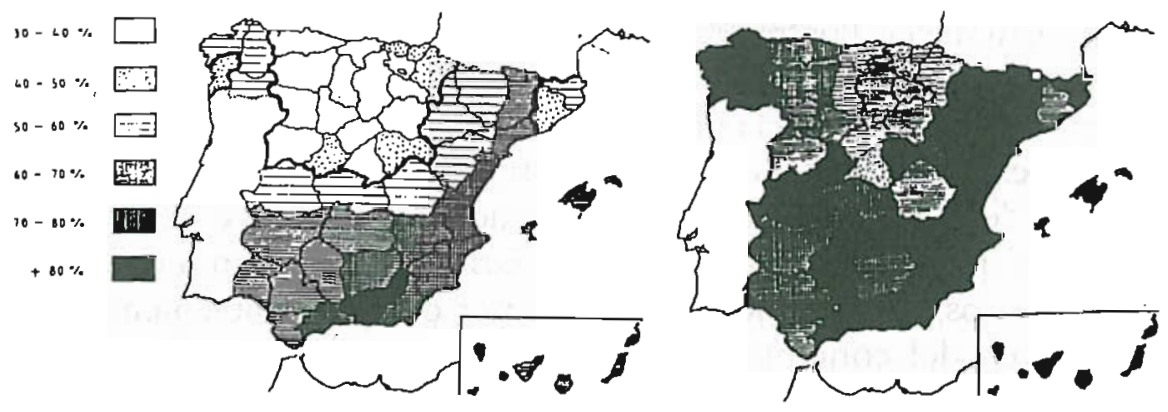

No parece necesario detallar cómo es la configuración de la geografía del analfabetismo, ya sea masculino o femenino, de la población española en 1877, puesto que nuestra explicación resultaría de nuevo reiterativa respecto a la efectuada en páginas anteriores. Baste señalar que, tanto en un caso como en otro, los mínimos se registran en las zonas ya tradicionales de mayor alfabetización: región centro y

(13) Sin embargo, sabemos, a ciencia cierta, que el interés por elevar el grado de instrucción de la mujer española se discutirá profusamente a partir de esos años en los círculos del intelectualismo más progresista del país. Así lo demuestran el gran número de ensayos y artículos, incluidos en el B.I.L.E., dedicados a un incipiente debate feminista. Por poner algunos ejemplos, véase: Ruiz Quevedo "La Asociación para la enseñanza de la mjuer", 1880, pp. 164-169 y 1881, pp. 97-101; Sela, A.: "La enseñanza de la mujer en Valencia", 1888, p. 109; Sela, A.: "Una nueva institución para la enseñanza de la mujer", 1889, p. 353; de Labra, R.M., "La rehabilitación de la mujer", 1891, pp. 138, 169, 187, 207, 222 y 235; Arenal, C.: "El trabajo de las mujeres", 1891, p. 314; Torres, R.: "Las profesiones de la mujer", 1893, 99. 33-65; Arenal, C.: "Estado actual de la mujer en España", 1895, 99. 311 y 338; Posada, A., "De un nuevo libro sobre el feminismo", 1899, 9. 107. 
norte, más foco barcelonés y gaditano; y los máximos, en los centros de analfabetismo conocidos, como Galicia, la mitad sur peninsular y, sobre todo, la región suroriental de la península, añadiendo también el estado de las islas Baleares y Canarias. Por otro lado, es perfectamente apreciable, visualmente, la diferencia cromática entre uno y otro mapa, entre uno y otro sexo, de manera que, una vez más, la imagen, como se suele decir, vale más que mil palabras, y se basta a sí misma para explicar el abismo que separa a hombres y mujeres en España en lo que a alfabetización se refiere. La misma conclusión podríamos apuntar para los mapas que especifican el comportamiento masculino y femenino en las capitales de provincia, sólo que, como ya hemos advertido en otra ocasión, en el espacio urbano se dan, lógicamente, las condiciones necesarias para reducir de forma considerable los porcentajes provinciales correspondientes.

\section{Analfabetismo masculino Analfabetismo femenino}

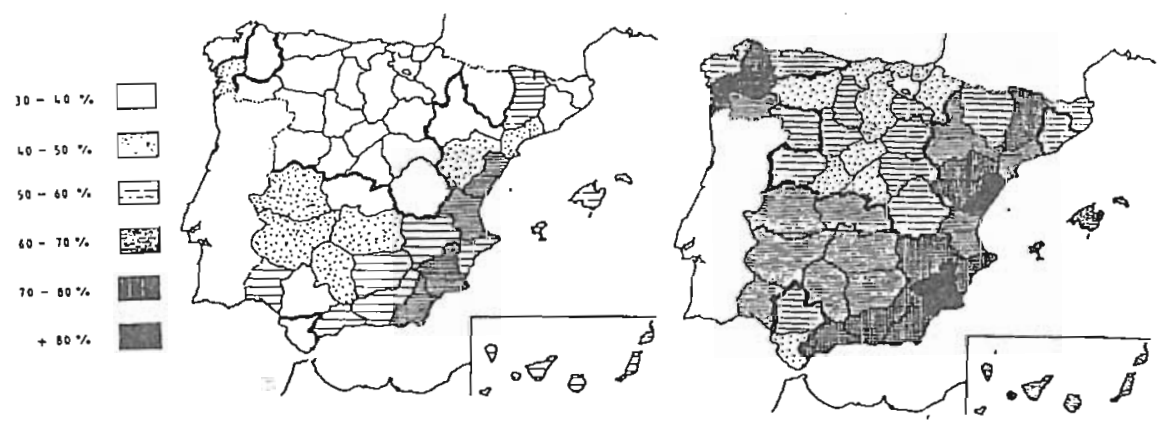

Quizá, lo más ilustrativo que quepa hacer en esta ocasión sea, precisamente, tratar de averiguar cuáles son los comportamientos anormales que se registran en la relación que se establece entre el índice de analfabetismo de ambos sexos, entendiendo como comportamiento anormal todo aquel que se separe del observado por las distintas medias nacionales, masculinas y femeninas, por provincias y por capitales. Considerando las provincias de Alava, Madrid, Navarra, Gui- 
púzcoa, Vizcaya, Cuenca y Cádiz (todas con porcentajes generales por debajo de la media nacional), de todas ellas se podrá decir que no sólo mantienen un nivel de analfabetismo entre los hombres de moderado a bajo sino que también son las provincias donde existe una cierta preocupación por la alfabetización de la mujer al poseer, al mismo tiempo, índices reducidos en este apartado. Sin embargo, provincias como Badajoz, Huelva, Sevilla, Jaén, Málaga, Granada, Almería, Albacete, Murcia y las tres levantinas, Alicante, Valencia y Castellón, mantienen unos valores que significan justamente todo lo contrario. Se trata, en està ocăsión, de los espaciôs que mantienèn un alto grado de analfabetismo, tanto masculino como femenino. Podemos decir que son las provincias que muestran una situación, siguiendo la expresión que hemos acuñado, de analfabetismo integral dentro de su conjunto poblacional. El caso de Granada, última de la lista, es suficientemente ilustrativo al superar, en ambos sexos, el $80 \%$ de censados mayores de siete años que no saben ni leer ni escribir. Existe otro bloque provincial que manifiesta un interés especial por la alfabetización masculina (no registra índices demasiado altos de analfabetismo), mientras que deja completamente de lado la instrucción de la población femenina que no sigue, ni de lejos, el grado de alfabetización de los varones. Es el caso, por ejemplo, de las cuatro provincias gallegas, que poseen un nivel moderado de analfabetismo en los hombres (La Coruña 55\%, Lugo $57 \%$, Orense $58 \%$ y Pontevedra $46 \%$ ), a la vez que sus cifras se disparan al observar el índice de desinstrucción de la mujer (La Coruña $86 \%$, Lugo $92 \%$, Orense $91 \%$ y Pontevedra 86\%). Esta misma desproporción entre sexos manifiestan las provincias que siguen, con equivalente interpretación a la de la región gallega: Burgos, Valladolid, Soria, La Rioja, León, Salamanca, Zamora, Avila, Asturias, Guadalajara, Gerona, Zaragoza, Cáceres, Huesca y Teruel. (Las provincias subrayadas poseen las diferencias máximas).

Lo que caracteriza al espacio urbano representado por los porcentajes adjudicados a las capitales de provincia es, en cuanto a la relación entre hombres y mujeres, la reducción drástica, de 19 a 6, de las ciudades con diferencias tajantes entre el estado de alfabetización masculina y femenina. Galicia se ratifica como el espacio más desproporcionado, más descompensado de todo el conjunto nacional, en esta comparación que hemos establecido entre los sexos. Pontevedra, Orense y Lugo mantienen diferencias sustanciales entre ambos (por ejemplo, Lugo presenta un analfabetismo masculino del $39 \%$ y femenino del $73 \%$ ). Dejando a un lado estas seis excepciones, el modo de 
vida urbano, por lo general, parece más propicio, no sólo para que la instrucción se generalice entre el grupo de los varones, sino que también parece ofrecer mejores oportunidades para la población femenina. El número de capitales de provincia que mantienen una diferencia proporcional, semejante al comportamiento medio del país, asciende a 27, más de la mitad del total, abundando en la interpretación anterior. Por otra parte, con niveles, en ambos casos, bajos o moderados se encuentran las siguientes ciudades: Vitoria, Madrid, Santander, Burgos, Segovia, Pamplona, Bilbao, San Sebastián, León, Avila y Cádiz. Son los centros donde se presta mayor atención a la instrucción de la mujer, al menos todo lo que cabe pensar dentro de las insuficiencias atribuibles a la práctica educativa de la España de esos años. Por último, con niveles parejos entre hombres y mujeres figuran también, pero esta vez reflejando un abandono general de la enseñanza, las capitales de Huelva, Jaén, Valencia, Santa Cruz de Tenerife y Almería.

Aquí concluimos lo que ha pretendido ser solamente una exposición general de lo que constituye nuestro panorama en materia de alfabetización a comienzos del último tercio del siglo XIX. Evidentemente, nos hemos movido bajo una doble intencionalidad a la hora de tratar exclusivamente la situación más epidérmica de la investigación que compete al historiador que pretenda abordar los temas educativos. Nos hemos valido fundamentalmente del método cuantitativo en nuestro trabajo, el más propicio, por otra parte, para indagar en esta específica parcela del saber histórico (14). Nos restaría, para dar sentido a los comportamientos regionales, urbanos y por sexos que hemos analizado, realizar las comparaciones oportunas buscando la razón última que justifique o explique cada estado descrito. Pero es aquí donde nuestro alcance cognitivo se movería bajo unas claras limitaciones, ya que la aplicación del método comparativo requiere del historiador el conocimiento profundo de cada realidad que pretende contrastar. La frase de R. Wittran sintetiza perfectamente lo que pretende-

(14) A comienzos de siglo, determinados estudiosos también acudían al análisis numérico: Luzuriaga, L.: El analfabetismo en España. Museo Pedagógico Nacional, Cosano, Madrid, 1926 (ed. revisada y aumentada, la primera se había publicado en 1919). Actualmente el método ha sido revisado convenientemente por Viñao Frago, A.: "Del analfabetismo a la alfabetización. Análisis de una mutación antropológica e historiográfica" (I) en Historia de la Educación, №3, 1984, Pp. 151-190 y (II) Historia de la Educación, N4, 1985, pP. 209-226. 
mos decir: "Sólo cuando se ha tenido en cuenta todo lo que conforma la individualidad de una situación histórica concreta se puede proceder con corrección a investigar qué es lo que tiene en común con otras situaciones aparentemente paralelas" (15). Siendo una tarea que por completo desborda nuestra capacidad, la de analizar, una por una, las condiciones en las que se desenvuelven los aspectos alfabetizadores de cada localidad, de cada región, estas páginas han pretendido, modestamente, movilizar el interés del trabajo local sobre estos aspectos, de modo que algún día podamos elaborar la síntesis final y definitiva que establezca la naturaleza de los factores que operan para que las diferencias interregionales que aquí quedan esbozadas sean, también, comprendidas.

(15) Cifr. Tiana Ferrer, A.: La Investigación Histórico Educativa Actual. Enfoques y Métodos, Cuadernos de la U.N.E.D. Madrid, 1988, p. 132. 